\title{
DEVELOPMENT OF SUPERCONDUCTING SOLDERED JOINTS BETWEEN BI-2212/AG WIRES
}

\author{
T. Mousavi, T. Davies, Z. Melhem, C.R.M. Grovenor and S.C. Speller
}

Abstract-Bi-2212/Ag superconducting wires are being considered as key conductor candidates for the development of high field magnets (up to $25 \mathrm{~T}$ ), but it is recognised that a reliable joining process between these wires is a critical challenge for most high-field applications. This work focuses on the design and testing of jointing processes between multifilamentary Bi-2212 wires to make persistent mode joints using soldering techniques. The ability of several different superconducting solder alloys from the PbBi and SnInBi systems to remove and replace the Ag matrix in fully reacted Bi-2212 wires has been explored. The SnInBi leadfree solder was found to be relatively effective at removing the Ag matrix, but does not make good contact with the Bi-2212 filaments. PbBi solder wets the Bi-2212 filaments more effectively, but is slower at removing the Ag matrix. Therefore, a two-stage process has been developed using molten Sn to first remove the $\mathrm{Ag}$ matrix followed by $\mathrm{PbBi}$ to replace the non-superconducting Sn. Joints made using this two-stage process have higher critical currents (133 A in self-field and $4 \mathrm{~K}$ ) than joints made using $\mathrm{PbBi}$ alone (120 $\mathrm{A}$ in self-field $4 \mathrm{~K}$ ).

Index Terms - Superconducting filaments and wires, Multifilamentary superconductors, Joints, Lead-free solder

\section{INTRODUCTION}

Bi-2212/Ag multifilamentary round wires are fabricated by a powder-in-tube technique [1]. The as-drawn wires are subsequently melt-processed using a complex heat treatment to form high critical current $\left(I_{c}\right)$ superconducting wire [2,3]. Since the wire is very brittle following this reaction process, magnets are wound before heat-treatment. Two approaches are being explored for fabricating persistent mode joints in these wires: melt-processing [4] and soldering [5]. In the melt-processing method, the Bi-2212 filaments of unreacted wires are exposed and joints are made between them using extra $\mathrm{Bi}-2212$ powder. The entire assembly is then melt-processed at the same time. In contrast, soldering methods can be used to form joints between wires that have already been reacted. This has the advantage of

Manuscript receipt and acceptance dates will be inserted here.

T. Mousavi, Tim Davies, Chris Grovenor and Susannah Speller are in the Centre for Applied Superconductivity in the Department of Materials, Oxford University, Parks Road, Oxford OX1 3PH, UK enabling joints to be made after the magnet assembly using techniques similar to those deployed in conventional $\mathrm{NbTi}$ magnets [6], allowing joints to be re-made if necessary. $\mathrm{PbBi}$ alloys are typically used to join NbTi wires owing to their relatively high $T_{c}$ and $B_{c 2}$ values [7]. Here we have investigated both standard $\mathrm{PbBi}$ solder and our best lead-free SnInBi solder alloy with composition close to a ternary eutectic, Sn-50In-35Bi [8] .

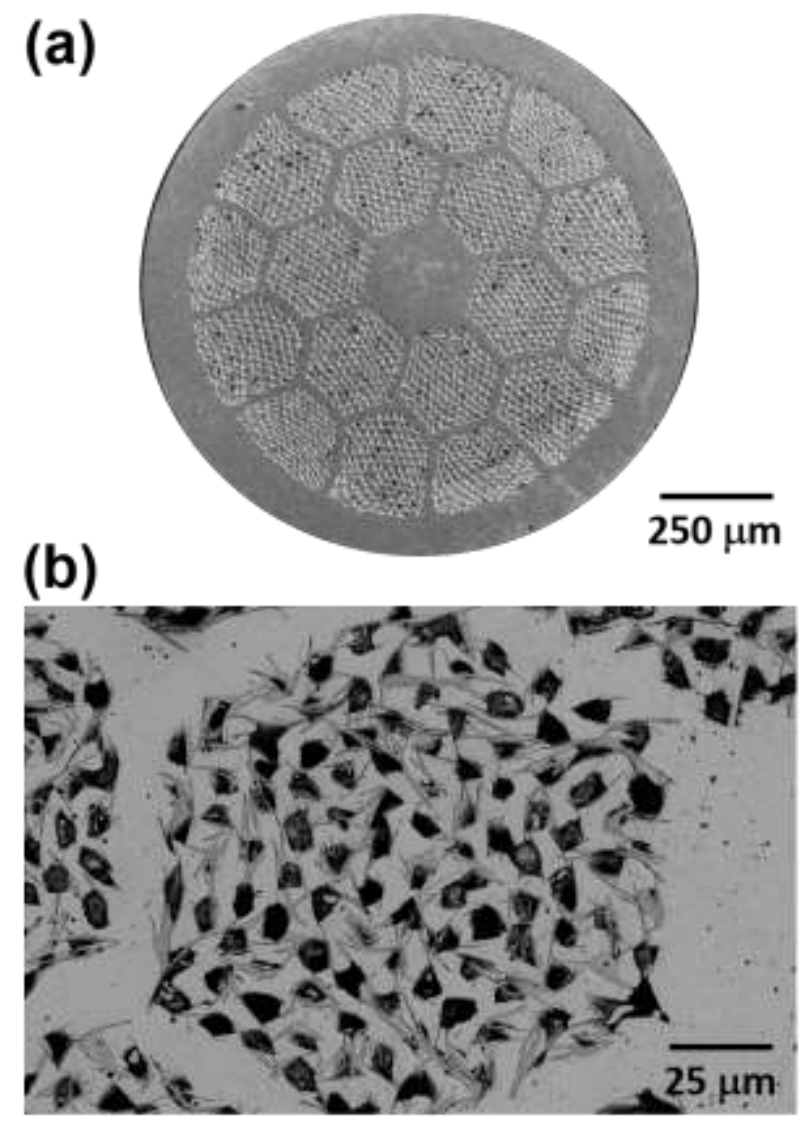

Figure 1: Backscattered electron images taken in a Zeiss EVO scanning electron microscope showing (a) the architecture of the Bi-2212/Ag strand, (b) the microstructure of a typical cross-section of a bundle of reacted $\mathrm{Bi}-2212 / \mathrm{Ag}$ wire.

Z. Melhem is with Oxford Instruments, Tubney Woods, Abingdon, OX13 5 QX, UK

Color versions of one or more of the figures in this paper are available online at http://ieeexplore.iee.org.

Digital Object Identifier will be inserted here upon acceptance. 
vided by OST. The as-received wires (with an $I_{C}$ value of $\sim 250 \mathrm{~A}$ at $4 \mathrm{~K}, 3 \mathrm{~T}$ ) were already exposed to the standard industrial heat treatment process. The architecture and the typical microstructure of the as-received wire are shown in Figure 1 a and $b$ respectively. Each wire incudes 18 bundles of $120 \mathrm{Bi}$ 2212 filaments within Ag matrix. The needlelike superconducting phase is seen to escape from the original filaments as a result of the heat treatment process.

In order to make direct bonds between the Bi-2212 filaments and the superconducting solder, the Ag matrix surrounding the filaments needs to be removed. The filaments could be exposed using an acidic etch prior to soldering, but the exposed filaments are easily damaged when the matrix has been removed. Here we instead investigate the ability of molten solder alloys to dissolve the $\mathrm{Ag}$ matrix, resulting in the replacement of the non-superconducting $\mathrm{Ag}$ matrix with superconducting solder without the need for a prior acid etch. To make a joint using this technique, lengths of reacted $\mathrm{Bi}-2212 / \mathrm{Ag}$ wire were immersed in molten solder baths held at $350{ }^{\circ} \mathrm{C}$ for 30 to 90 minutes. Then the immersed lengths were held next to each other with overlaps of $5 \mathrm{~mm}$ and $10 \mathrm{~mm}$. A small amount of molten solder was finally dropped in the overlap area to make joints.

\section{B. Mapping the solder interactions}

Figure 2 shows energy dispersive $\mathrm{x}$-ray (EDX) phase maps of wire cross-sections taken using a Zeiss EVO scanning electron microscope (SEM). After 30 minutes immersion in SnInBi, the outer layer of $\mathrm{Ag}$ has been successfully removed and the solder has started to infiltrate between the outer bundles of the Bi-2212 filaments. Increasing the immersion time to 90 minutes results in the solder penetrating almost to the centre of the wire. However, the solder does not seem to wet the Bi-2212 filaments very effectively, resulting in gaps between the solder and the filaments which appear black in Figure 2 (b). In contrast, $\mathrm{PbBi}$ solder does not dissolve the $\mathrm{Ag}$ matrix as quickly as $\mathrm{SnInBi}$, so that the $\mathrm{PbBi}$ solder does not fully penetrate the inner filament bundles even after 90 minutes. However, $\mathrm{PbBi}$ solder appears to form better interfaces with the Bi-2212 filaments, as can be seen by the high resolution EDX maps in Figure 3.

Since the PbBi solder is not very effective at infiltrating the $\mathrm{Bi}-2212$ wires, a two-stage process was developed using nonsuperconducting pure $\mathrm{Sn}$ to initially remove the $\mathrm{Ag}$ matrix. As seen in Figure 2 (e), molten Sn is very effective, infiltrating the entire wire within 60 minutes. The Sn matrix is subsequently replaced by superconducting $\mathrm{PbBi}$ alloy in a second process. This resulted in much better infiltration of $\mathrm{PbBi}$ into the core of the wire compared with the single stage $\mathrm{PbBi}$ process. However, high resolution EDX analysis revealed a residual Sn-O interfacial layer about $500 \mathrm{~nm}$ thick around each filament Figure 4. This layer is likely to act as a barrier to superconducting current transfer, potentially limiting joint performance. Further (a) SnInBi-30 mins

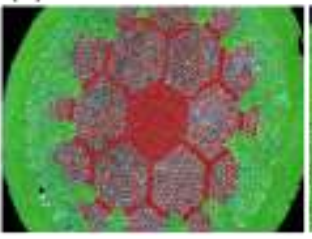

(d) SnlnBi-90 mins

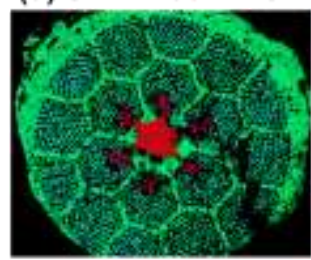

$500 \mu \mathrm{m}$ (b) $\mathrm{PbBi}-30$ mins

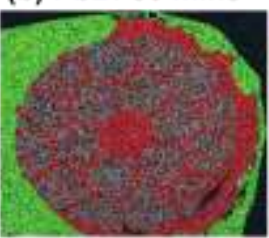

(c) Sn-60 mins

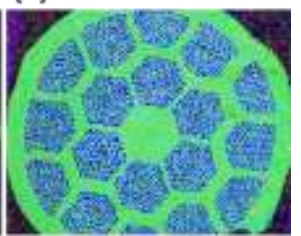

(e) PbBi-90 mins
Sn-60 mins +

PbBi-60 mins
Figure 2: SEM/EDX phase maps showing infiltration of solder after immersion in SnInBi alloy for (a) 30 mins and (b) 90 mins, PbBi solder for (c) 30 mins and (d) 90 mins, (e) pure $\mathrm{Sn}$ for $60 \mathrm{mins}$, and (f) $\mathrm{Sn}$ for 60 mins followed by $\mathrm{PbBi}$ for $60 \mathrm{mins}$.
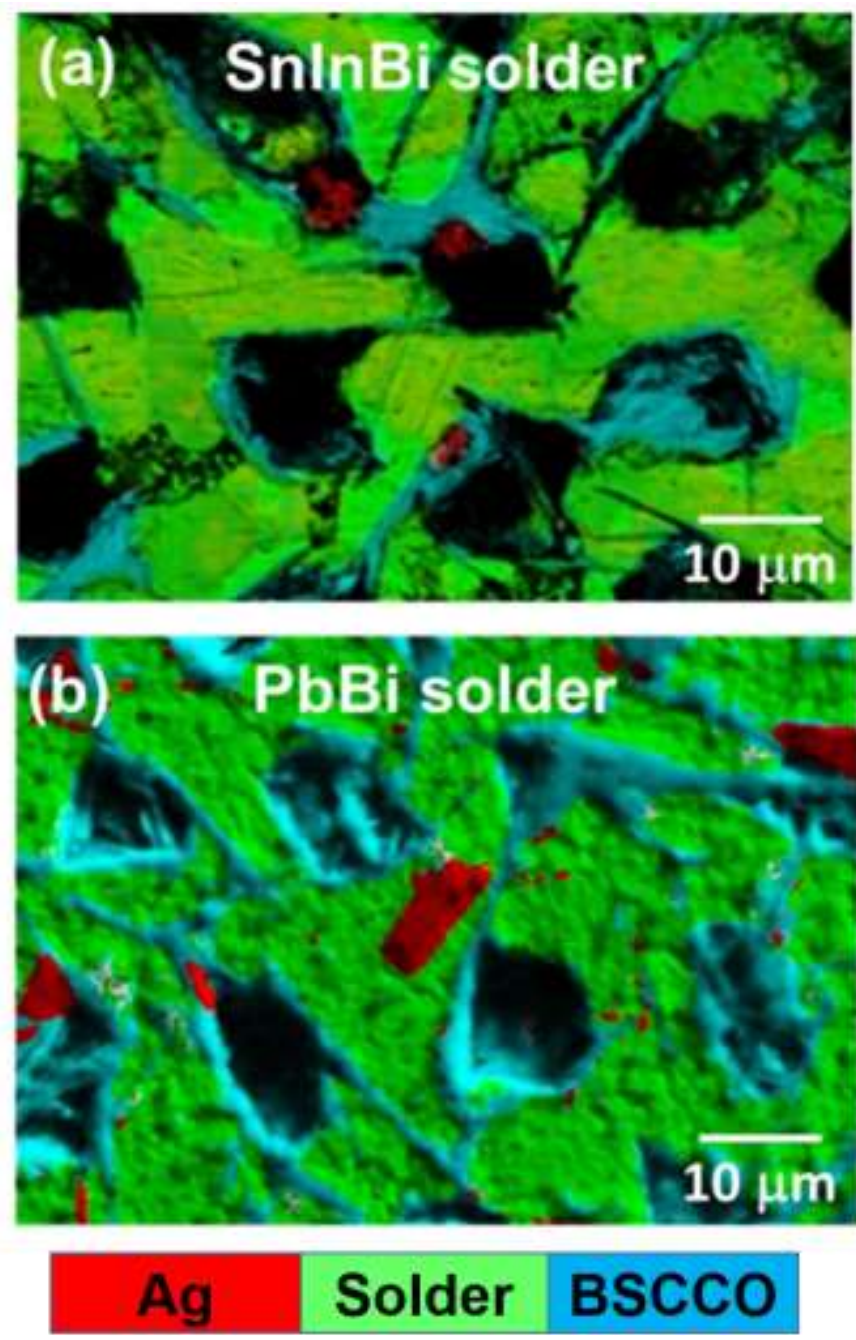

Figure 3: SEM/EDX maps for (a) SnInBi and (b) PbBi solder. Black cracks are seen around the $\mathrm{Bi}-2212 / \mathrm{SnInBi}$ interfaces. These cracks are not seen when $\mathrm{PbBi}$ solder is used. Some residual Ag alloy particles remain in the solder. 
experimental work is needed to explore whether the formation of this undesirable reaction layer can be minimized by optimization of the tinning temperature and time.
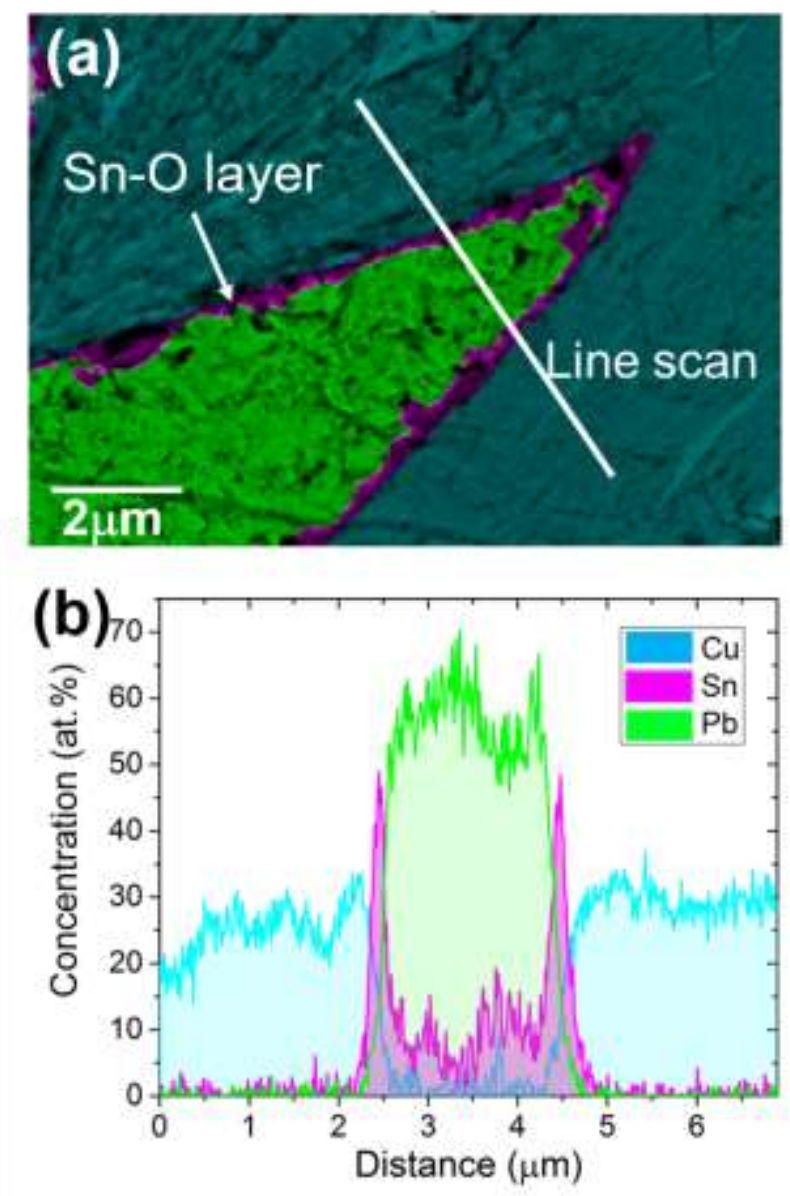

Figure 4: High resolution EDX phase map taken in a Zeiss Merlin FEGSEM shows a thin Sn-O interfacial layer. The line scan shows the layer is about 500 nm thick.

\section{JOINT PROPERTIES}

The superconducting properties of joints made using the single stage $(\mathrm{PbBi})$ process and the two stage $(\mathrm{Sn}+\mathrm{PbBi})$ process have been compared.

Figure 5 shows low resolution EDX maps of the joint crosssection, confirming that the two-stage process is significantly more effective at removing the Ag matrix. The superconducting performance of joints have been measured at $4 \mathrm{~K}$ using 4-terminal transport measurements on short straight sections of wire containing a single lap-style joint in applied magnetic fields of 0 to $1.8 \mathrm{~T}$ in a direction parallel to the wire axis. As shown in Figure 6, a $10 \mathrm{~mm}$ long joint fabricated using the single stage $\mathrm{PbBi}$ solder process ( $\mathrm{PbBi}$ joint) is found to have comparable $I_{c}$ performance to a $5 \mathrm{~mm}$ long joint fabricated using

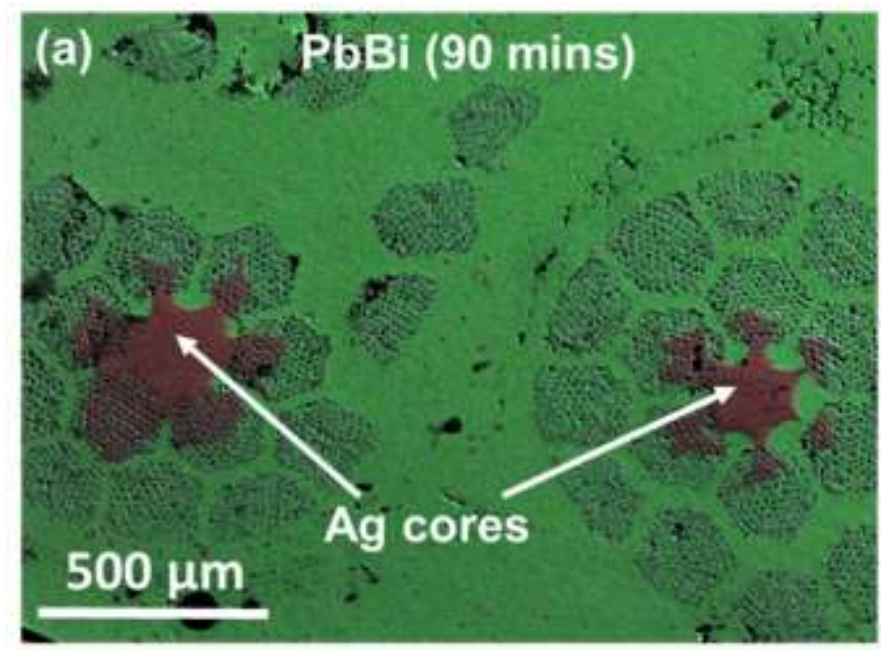

\section{(b) Sn (60 mins) $+\mathrm{PbBi}(60$ mins $)$}

\section{$500 \mu \mathrm{m}$}

Figure 5: EDX/SEM phase maps of PbBi cross-sections of joints made by (a) single stage 90 minute $\mathrm{PbBi}$ solder process, and (b) two stage process using $\mathrm{Sn}$ for 60 minutes followed by $\mathrm{PbBi}$ for 60 minutes.

the two stage process ( $\mathrm{Sn}+\mathrm{PbBi}$ joint). Using a standard electric field criterion of $100 \mu \mathrm{V} \mathrm{m}^{-1}$, the $\mathrm{PbBi}$ joint and $\mathrm{Sn}+\mathrm{PbBi}$ joints had $I_{c}$ values of $120 \mathrm{~A}$ and $133 \mathrm{~A}$ in self-field, respectively. In $1 \mathrm{~T}$ field, the $I_{c}$ values dropped to $40 \mathrm{~A}$ and $49 \mathrm{~A}$, respectively. Under these conditions, the critical current density of the $\mathrm{PbBi}$ solder will be significantly lower as the upper critical field for this alloy is $1.77 \mathrm{~T}$ at $4 \mathrm{~K}$ [7]. SnInBi alloy has a $B_{c 2}$ value that is too low (about $0.2 \mathrm{~T}$ at $4 \mathrm{~K}$ ) to use in this application, so the perfomance of joints made using this solder are not reported here [8]. 

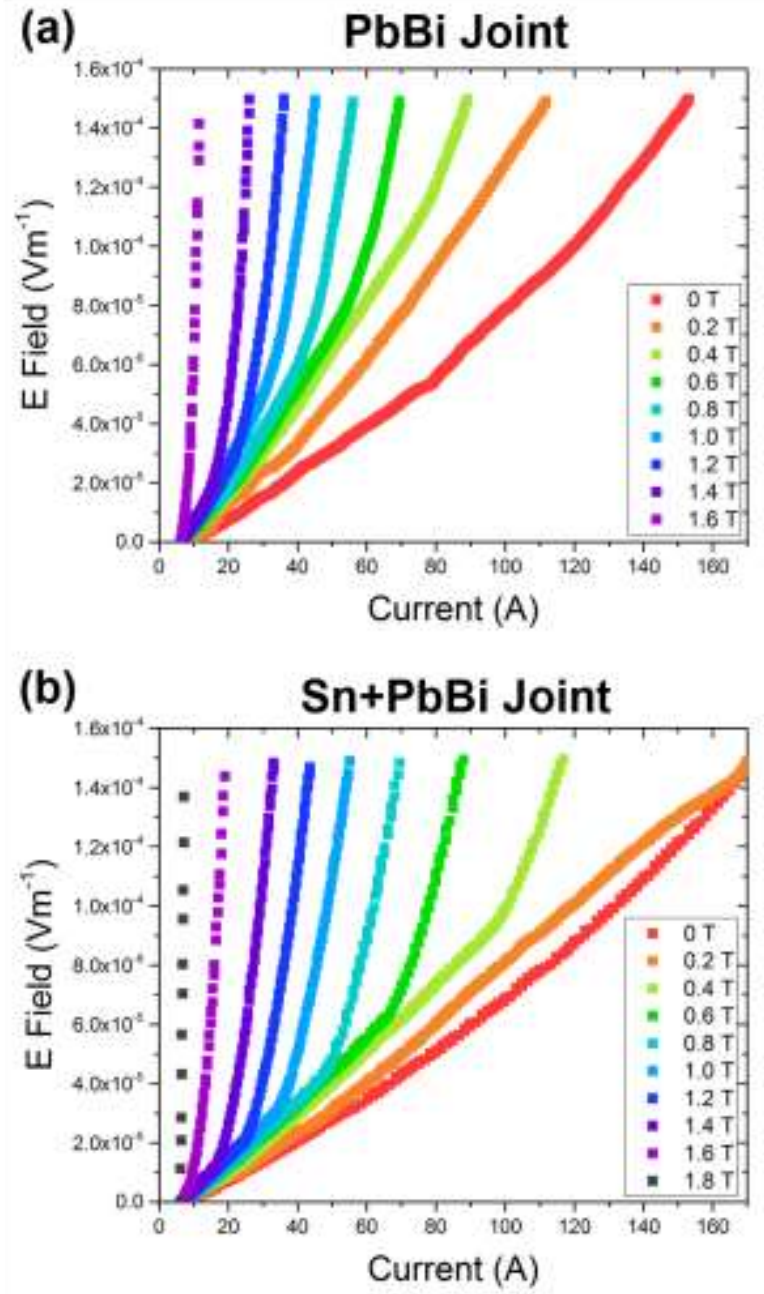

Figure 6: Transport measurements of PbBi joints made by (a) single stage process (10 $\mathrm{mm}$ long joint), and (b) two stage process (5 $\mathrm{mm}$ long joint).

\section{CONCLUSIONS}

Soldering techniques have been investigated for fabricating superconducting lap joints between reacted $\mathrm{Bi}-2212$ wires. $\mathrm{PbBi}$ solder is found to form good quality interfaces with $\mathrm{Bi}$ 2212 filaments, whereas lead-free SnInBi solder alloy does not appear to wet Bi-2212 effectively. However, $\mathrm{PbBi}$ solder does not remove the Ag matrix from the wires very effectively, so a two stage process has been developed initially using pure molten $\mathrm{Sn}$ to remove the $\mathrm{Ag}$ matrix followed by immersion in $\mathrm{PbBi}$ to replace the $\mathrm{Sn}$. This significantly improves the infiltration of $\mathrm{PbBi}$ to the centre of the $\mathrm{Bi}-2212$ wires. However, high resolution EDX analysis has revealed that a thin layer of $\mathrm{Sn}-\mathrm{O}$ remains at the interface between the $\mathrm{Bi}-2212$ filaments and the solder. Despite the presence of this non-superconducting layer around all the filaments, the performance of joints fabricated using the two-stage process were superior, with a $5 \mathrm{~mm}$ long joint having better performance than a $10 \mathrm{~mm}$ joint made using the single stage process. As can be seen in Figure 6 , the joints here have a finite resistance even at low currents and their critical current density is much lower than the parent wire ( 250 A at $4 \mathrm{~K}, 3 \mathrm{~T}$ ), so further improvements are required to enable persistent mode operation for NMR/MRI applications.

\section{ACKNOWLEDGMENTS}

The authors would like to thank the Oxfordshire Local Enterprise Partnership for providing funding for the Oxford Centre for Applied Superconductivity. Thanks also to M. Bristow in the Physics Department, University of Oxford for assistance with the high current testing and Y. Huang at Bruker-OST for providing wire samples. Data in support of this paper is accessible on the Oxford Research Archive (www.ora.ox.ac.uk).

\section{REFERENCES}

[1] K. Heine, J. Tenbrink, M. Thoner, "High field critical current densities in $\mathrm{Bi}_{2} \mathrm{Sr}_{2} \mathrm{Ca}_{1} \mathrm{Cu}_{2} \mathrm{O}_{8+\mathrm{x}} / \mathrm{Ag}$ wires", Appl. Phys. Lett. 55(23) (1989) 2441.

[2] E.E. Hellstrom, "Important considerations for processing Bi-based hightemperature superconducting tapes and films for bulk applications", $M R S$ Bull. 17 (1992) 45

[3] A. Matsumoto, H. Kitaguchi, H. Kumakura, J. Nishioka and T. Hasegawa, "Improvement of the microstructure and critical current densities of Bi-2212 round wires with a precisely controlled heat treatment", Supercond. Sci. Technol. 17 (2004) 989

[4] P. Chen, U. P. Trociewitz, D. S. Davis, E. S. Bosque, D. K. Hilton, Y. Kim, D. V. Abraimov, W. L. Starch, J. Jiang, E. E. Hellstrom, D. C. Larbalestier, "Development of a persistent superconducting joint between Bi-2212/Ag-alloy multifilamentary round wire", Supercond. Sci. Technol. 30 (2016) 025020

[5] S. Y. Oh, H. R. Kim, Y. H. Jeong, O. B. Hyun, C. J. Kim, "Joining of Bi2212 high- $T_{c}$ superconductors and metals using indium solder", Physica C 463 (2007) 464

[6] G. D. Brittles, T. Mousavi, C. R. M. Grovenor, C. Aksoy and S. C. Speller, "Persistent current joints between technological superconductors", Supercond. Sci. Technol., 28 (2015) 093001

[7] J. E. Evetts and J. M. Wade, "Superconducting properties and the phase diagrams of the $\mathrm{Pb}-\mathrm{Bi}$ and $\mathrm{Pb}-\mathrm{In}$ alloy systems", J. Phys. Chem. Solids 31 (1970) 973

[8] T. Mousavi, C. Aksoy, C. R. M. Grovenor and S. C. Speller, "Microstructure and superconducting properties of Sn-In and Sn-In-Bi alloys as Pb-free superconducting solders", Supercond. Sci. Technol. 29 (2016) 015012 